\title{
The heterocyclic compound Tempol inhibits the growth of cancer cells by interfering with glutamine metabolism
}

\author{
Shuangyan Ye', Pengfei Xu' ${ }^{1}$, Mengqiu Huang ${ }^{1}$, Xi Chen' ${ }^{1}$, Sisi Zeng ${ }^{1}$, Qianli Wang ${ }^{1}$, Jianping Chen', Keyi Li', \\ Wenwen Gao', Ruiyuan Liu', Jingxian Liu', Yihao Shao ${ }^{3}$, Hui Zhang ${ }^{4}$, Yang Xu', Qianbing Zhang ${ }^{1}$, Zhuo Zhong ${ }^{5}$, \\ Zibo Wei ${ }^{6}$, Jiale Wang ${ }^{6}$, Bingtao Hao', Wenhua Huang ${ }^{7,8}$ and Qiuzhen Liu,
}

\begin{abstract}
Tempol (4-hydroxy-2,2,6,6-Tetramethylpiperidine-1-oxyl, TPL), a nitroxide compound, inhibits proliferation and increases the vulnerability of cancer cells to apoptosis induced by cytotoxic agents. However, the molecular mechanism of TPL inhibiting cancer cell proliferation has not been fully understood. In this study, we evaluated the metabolic effect of TPL on cancer cells and explored its cancer therapeutic potential. Extracellular flow assays showed that TPL inhibited cellular basal and maximal oxygen consumption rates of mitochondrial. ${ }^{13} \mathrm{C}$ metabolic flux analysis showed that TPL treatment had minimal effect on glycolysis. However, we found that TPL inhibits glutamine metabolism by interfering with the oxidative tricarboxylic acid cycle (TCA) process and reductive glutamine process. We found that the inhibitory effect of TPL on metabolism occurs mainly on the step from citrate to a-ketoglutarate or vice versa. We also found that activity of isocitrate dehydrogenase IDH1 and IDH2, the key enzymes in TCA, were inhibited by TPL treatment. In xenograft mouse model, TPL treatment reduced tumor growth by inhibiting cellular proliferation of xenograft tumors. Thus, we provided a mechanism of TPL inhibiting cancer cell proliferation by interfering with glutamine utilization that is important for survival and proliferation of cancer cells. The study may help the development of a therapeutic strategy of TPL combined with other anticancer medicines.
\end{abstract}

\section{Introduction}

Tempol (4-hydroxy-2,2,6,6-Tetramethylpiperidine-1oxyl, TPL), a stable nitroxide radical used as a contrast agent in magnetic resonance imaging ${ }^{1}$, has been reported to induce cellular apoptosis in many types of cancer cells $^{2-4}$. The toxicity effect of TPL presents a

Correspondence: Bingtao Hao (haobt123@smu.edu.cn) or

Wenhua Huang (huangwenhua2009@139.com) or

Qiuzhen Liu (liuqiuzhen@126.com)

${ }^{1}$ Cancer Research Institute, Guangdong Provincial Key Laboratory of Cancer Immunotherapy, Guangzhou Key Laboratory of Tumor Immunology Research, School of Basic Medical Sciences, Southern Medical University, Guangzhou, China

${ }^{2}$ School of Biomedical Engineering, Southern Medical University, Guangzhou, China

Full list of author information is available at the end of the article These authors contributed equally: Shuangyan Ye, Pengfei Xu Edited by A. Stephanou concentration-dependent manner and is preferential to target cancer cells ${ }^{5}$. TPL induces the expression of the cycling-dependent kinase inhibitor p21waf1/cip1, and activates caspase- 3 and $\mathrm{Bax} / \mathrm{Bcl}-2$ pathway ${ }^{2,6}$. And the cytotoxicity of TPL also depends on the induction of cellular oxidative stress ${ }^{7,8}$. It is reported that high concentration or prolonged TPL treatment increases cellular ROS level, while low concentration of TPL reduces ROS (ref. ${ }^{9}$ ), which is related to the fact that TPL can switch between oxidation and reduction states, hydroxylamine and oxoammonium cation ${ }^{10}$. The dosage of TPL used in vivo usually mimics the MnSOD activity and plays an anti-oxidative role ${ }^{11,12}$. Some studies showed that TPL functions as an antioxidant and improves the performance in animal models of many diseases, including hypertension $^{13}$, diabetes ${ }^{14}$, diabetic kidney disease ${ }^{15}$,

\section{(-) The Author(s) 2020}

(c) (i) Open Access This article is licensed under a Creative Commons Attribution 4.0 International License, which permits use, sharing, adaptation, distribution and reproduction cc) in any medium or format, as long as you give appropriate credit to the original author(s) and the source, provide a link to the Creative Commons license, and indicate if changes were made. The images or other third party material in this article are included in the article's Creative Commons license, unless indicated otherwise in a credit line to the material. If material is not included in the article's Creative Commons license and your intended use is not permitted by statutory regulation or exceeds the permitted use, you will need to obtain permission directly from the copyright holder. To view a copy of this license, visit http://creativecommons.org/licenses/by/4.0/. 
neurodegenerative ${ }^{16,17}$, and ischemia-reperfusion injury ${ }^{18}$. Therefore, the concentration of TPL in vivo is usually too low to induce oxidative stress. So whether the in vivo TPL treatment inhibits tumor growth is still a question.

A common feature of cancer cell metabolism is the ability to use intermediates of glycolysis/tricarboxylic acid (TCA) cycle for biosynthesis and NADPH production, which are essential for building new biomass ${ }^{19,20}$. It's known that cancer cells have a high-rate glucose uptake and metabolism to meet cell growth and viability. It has been reported that antioxidant, vitamin $C$, can disturb glucose metabolism in primary rat adipocytes ${ }^{21}$. But whether the antitumor effect of TPL is associated with the regulation of glucose metabolism is yet to be determined.

Aside from glucose, recent studies showed that amino acids, especially glutamine, are also involved in the biosynthesis in cancer cells ${ }^{22,23}$. Many types of cancer cells display elevated glutamine flux, and even some cancer cell lines exhibit glutamine addiction ${ }^{24,25}$. Glutamine metabolism occurs mainly in mitochondrial. Studies suggest that TPL is accumulated in mitochondrial and interacts with transition metal ions in catalytic centers of enzyme complexes associated with the respiratory chain complexes (complexes I, II, and IV), which impairs OXPHOS and reduces intracellular glutathione pool, and then induces oxidative stress and cellular apoptosis ${ }^{7,26-28}$. But whether TPL is involved in glutamine metabolism remains unclear.

Here, we characterized the antiproliferative effects of TPL with in vitro and in vivo experiments, and elucidated the metabolic mechanisms involved in glucose and glutamine metabolism alteration in TPL-treated ovarian cancer cells by ${ }^{13} \mathrm{C}$ metabolic flux analysis. We found that TPL treatment interfered with the glutamine utilization in cancer cells. It was described below.

\section{Materials and methods}

\section{Chemicals and reagents}

TPL was purchased from Adamas Reagent Co., Ltd. (Shanghai, Chian). 3-(4,5-Dimethylthiazol-2-yl)-2,5diphenyltetrazoliumbromide (MTT) and dimethyl sulfoxide (DMSO) were purchased from Sigma (St. Louis, MO, USA). Other reagents were obtained from the following companies: methanol (Kermel, Tianjin, China), chloroform, hexane, and pyridine (Aladdin, Shanghai, China), $\left[{ }^{13} \mathrm{C}-\mathrm{U}_{6}\right]$ glucose, $\left[{ }^{13} \mathrm{C}-\mathrm{U}_{5}\right]$ glutamine (Cambridge, Boston, MA, USA), 2\% methoxylamine hydrochloride, $\mathrm{N}$ tert-butyldimethylsily-N-methyltrifluoroacetamide (MTBSTFA), tert-butyldimethylchlorosilane (tBDMS; Sigma, St. Louis, MO, USA), glucose-free DMEM medium, and glutamine-free RPMI-1640 medium (Gibco, Waltham, MA, USA). Glucose, oligomycin, 2-DG, carbonyl cyanide 4-(trifluoromethoxy)phenylhydrazone (FCCP), and rotenone-antimycin A (Agilent Technologies, Santa Clara, CA, USA). L-glutamine and pyruvate (Thermo Fisher Scientific, Waltham, MA, USA).

\section{Cell culture}

The human ovarian cancer line SKOV3 were obtained from ATCC. SKOV3 was maintained in RPMI-1640 medium with $10 \%$ FBS and $1 \%$ antibiotics mixture. The cells were maintained under standard culture conditions at $37^{\circ} \mathrm{C}$ with a $5 \% \mathrm{CO}_{2}$ atmosphere in a humidified incubator unless otherwise mentioned. And cells were continuously cultured in without pen/strep exposure.

\section{Cytotoxicity assays}

MTT assays were performed to determine the viability of cell lines. Cells were seeded onto 96-well plates, allowed to attach, and grow for $12-24 \mathrm{~h}$, and subsequently exposed to TPL in different concentrations or not. After $48 \mathrm{~h}, 100 \mu \mathrm{l}$ of MTT $(0.5 \mathrm{mg} / \mathrm{ml}$ in medium $)$ was added to cells for $3 \mathrm{~h}$ at $37^{\circ} \mathrm{C}$. Moved MTT and slightly rinsed twice with PBS. The formazan crystals then were solubilized in $100 \mu \mathrm{l}$ of DMSO. The samples were shaken for $15 \mathrm{~min}$ at $37^{\circ} \mathrm{C}$ and the absorbance was read at $490 \mathrm{~nm}$ using Bio-Tek multi-mode reader.

\section{ROS level analysis}

To detect the ROS level in culture cell, $35 \times 10^{4}$ cells/ well were seeded onto six-well plates, and subsequently exposed to TPL in different concentrations for $24 \mathrm{~h}$ after incubating at $37{ }^{\circ} \mathrm{C}$ for $12 \mathrm{~h}$. Collected and washed cells twice with normal temperature PBS for ROS determination. The determination of ROS was detected using ROS Assay Kit according to the manufacturer's instructions (Beyotime, Shanghai, China). Briefly, $1 \times 10^{7}$ cells $/ \mathrm{ml}$ were suspended on serum-free medium containing $10 \mu \mathrm{m} / \mathrm{ml}$ DCFA-DA and incubated at $37^{\circ} \mathrm{C}$ in the dark for $20 \mathrm{~min}$. Unincorporated DCFA-DA was moved by washing the cells with phenol red free medium. Then cells were resuspended with PBS, and immediately subjected to flow cytometric analysis. The experiments were performed at least three times.

\section{$\mathrm{NAD}^{+}$and $\mathrm{NADH}$ measurement}

To assess the $\mathrm{NAD}^{+}$and NADH, $35 \times 10^{4}$ cells/well were seeded onto six-well plates. The amount of $\mathrm{NAD}^{+}$ and $\mathrm{NADH}$ was measured using $\mathrm{NAD}^{+} / \mathrm{NADH}$ Assay Kit (Beyotime, Shanghai, China), according to the manufacturer's protocol. Before detecting $\mathrm{NADH}$, samples were heated in water bath to $60^{\circ} \mathrm{C}$ for $30 \mathrm{~min}$ to decompose $\mathrm{NAD}^{+}$. Then the decomposed sample was reacted and measured at $450 \mathrm{~nm}$ using Bio-Tek multi-mode reader. The content of NADH in the sample can be calculated from standard curve. 


\section{Glucose uptake analysis}

To assess the glucose uptake, $6 \times 10^{4}$ cells/well were seeded onto 12-well plates. After cells attached on the wells, exchanged the medium into medium containing $0.8 \mathrm{mM}$ TPL or not to culture for $24 \mathrm{~h}$. The measurement of glucose was detected using Glucose Colorimetric Assay Kit II according to the manufacturer's instructions (BioVision, SanFrancisco, CA, USA). Briefly, $2 \mu \mathrm{l}$ of cell culture supernatant was added to $48 \mu \mathrm{l}$ of glucose assay buffer in 96-well plate, and mixed well. Added $50 \mu \mathrm{l}$ of reaction mix, containing $46 \mu \mathrm{l}$ glucose assay buffer, $2 \mu \mathrm{l}$ glucose enzyme mix, and $2 \mu$ l glucose substrate mix, to each well containing the test samples. Incubated the reaction for $30 \mathrm{~min}$ at room temperature (RT) that protected from light. Measured absorbance at $450 \mathrm{~nm}$ in a microplate reader, the glucose concentrations in supernatant can be calculated from standard curve.

\section{Metabolite extraction}

For analysis of metabolism, $40 \times 10^{4}$ cells/well SKOV3 cells were seeded onto a six-well plate with basal medium in at least three repeats for $12 \mathrm{~h}$, allowing cells attaching enough. Then steady-state labeling of organic was accomplished by changing the culture medium into glucose-free DMEM medium or glutamine-free RPMI1640 medium containing appropriate tracer, including $11 \mathrm{mmol} / \mathrm{l}\left[\mathrm{U}_{-}{ }^{13} \mathrm{C}_{6}\right]$ glucose or $2 \mathrm{mmol} / \mathrm{l}\left[\mathrm{U}_{-}{ }^{13} \mathrm{C}_{5}\right]$ glutamine, with or without $0.8 \mathrm{mM}$ TPL for $24 \mathrm{~h}$.

At the end of culture, cells were rinsed twice with normal temperature $0.9 \% \mathrm{NaCl}$ solution, quenched with $500 \mu \mathrm{l} /$ well $-80^{\circ} \mathrm{C}$ methanol. One minute later, $200 \mu \mathrm{l}$ of ice-cold $5 \mu \mathrm{g} / \mathrm{ml}$ norvaline was added to each well and cells were collected in $1.5 \mathrm{ml}$ Eppendorf tubes by scraping with a pipette. Then $500 \mu \mathrm{l}$ of $-20^{\circ} \mathrm{C}$ chloroform was added to each tube followed by vortexing for $15-20 \mathrm{~min}$, and centrifugation at $14,000 \times g$ for $10 \mathrm{~min}$ at $-4{ }^{\circ} \mathrm{C}$. The upper aqueous phase and the lower organic layer were transferred to the fresh tube and exsiccated of airflow, respectively. These dried sample can be stored at $-20^{\circ} \mathrm{C}$.

\section{Metabolite derivation}

Derivation should be done within $24 \mathrm{~h}$ before detection. Polar metabolites were derivatized to form methoxime-tBDMS derivatives by dissolved upper dried metabolites with $20 \mu \mathrm{l}$ of $2 \%(\mathrm{~m} / \mathrm{v})$ methoxylamine hydrochloride in pyridine and incubating at $37^{\circ} \mathrm{C}$ for $60 \mathrm{~min}$. Samples were then silylated by addition of $100 \mu \mathrm{l}$ of MTBSTFA with $1 \%$ tBDMS and incubated at $45^{\circ} \mathrm{C}$ for $30 \mathrm{~min}$. Transferred to glass GC vials for analysis.

\section{GC-MS analysis}

Derivatized metabolites were analyzed using a Thermo GC 1300 connecting to a Thermo MS ISQ. For polar metabolites, the $\mathrm{GC}$ oven temperature was held at $100^{\circ} \mathrm{C}$ for $2 \mathrm{~min}$, and increased to $255^{\circ} \mathrm{C}$ at $3.5^{\circ} \mathrm{C} / \mathrm{min}$ followed by increasing to $320^{\circ} \mathrm{C}$ at $15^{\circ} \mathrm{C} / \mathrm{min}$ and held for $3 \mathrm{~min}$. Electron impact ionization was operated with the MS scanning over the range $100-650 \mathrm{~m} / \mathrm{z}$.

\section{Determination of extracelluler acidification and oxygen consumption}

Then measurement of extracelluler acidification rate (ECAR) and oxygen consumption rate (OCR) were detected by XF96 Extracellular Flow analyzer (Seahorse Bioscience, Billerica, Ma, USA) in real time. Briefly, $1 \times$ $10^{4}$ cells/well SKOV3 cells were cultured in custom XF96 microplates. After cell attaching well $(\sim 12 \mathrm{~h})$, the media was replaced with $80 \mu \mathrm{l}$ of RPMI-1640 media containing indicated concentration of TPL for $24 \mathrm{~h}$. Before measurement, non-buffer XF assay medium was preheated to $37^{\circ} \mathrm{C}$, and cells were washed with the assay medium twice. Immersed in $180 \mu \mathrm{l}$ non-buffered medium and incubated in the $37^{\circ} \mathrm{C}$, and non- $\mathrm{CO}_{2}$ incubator for $1 \mathrm{~h}$. All experiments were performed at $37^{\circ} \mathrm{C}$. In order to eliminate the effect of the cell proliferation rate, determined the concentrations of protein in each well after finishing the assay and then normalized either OCR or ECAR by dividing the concentration. Cellular viabilities that were determined after assay were nearly indistinguishable regardless of $24 \mathrm{~h}$ TPL exposure or not. For measurement of ECAR, glucose $(10 \mathrm{mM})$, oligomycin $(1 \mu \mathrm{M})$, and 2-DG $(50 \mathrm{mM})$ were added at a specified point in time. For measurement of OCR, oligomycin $(1 \mu \mathrm{M})$, FCCP $(0.5 \mu \mathrm{M})$, and rotenoneantimycin $\mathrm{A}(0.5 \mu \mathrm{M})$ were added at a specified point in time. The bracketed concentrations of drugs are the final concentrations.

\section{CCK 8 assay}

A total of 3000 cells/well SKOV3 cells were cultured on 96-well plate. After cells attached on the wells, the medium was exchanged into glutamine-free RPMI-1640 medium, glutamine-free RPMI-1640 medium containing $0.8 \mathrm{mM}$ TPL, glutamine-free RPMI-1640 medium containing $2 \mathrm{mM} \alpha$-ketoglutarate, glutamine-free RPMI-1640 medium containing $2 \mathrm{mM} \alpha$-ketoglutarate, and $0.8 \mathrm{mM}$ TPL to culture for $48 \mathrm{~h}$. Washed cells with glutamine-free RPMI-1640 medium twice, following by adding $100 \mu \mathrm{l}$ glutamine-free RPMI-1640 medium to each well. Then $10 \mu \mathrm{l}$ of CCK8 was added to each well and the cells were incubated at $37^{\circ} \mathrm{C}$ for $2 \mathrm{~h}$ away from light. The absorbance was read at $450 \mathrm{~nm}$ using Bio-Tek multi-mode reader.

\section{Molecular modeling}

Autodock vina docking software, offered free by The Scripps Research Institute (http://vina.scripps.edu/), was used to predict the binding mode. The three-dimensional (3D) structure of TPL was obtained from PubChem 
database (https://pubchemdocs.ncbi.nlm.nih.gov/). The resulting structures were saved in 3D SDF format. The crystallographic structures of isocitrate dehydrogenase 1 (IDH1) (PDB ID: 6ADG) and IDH2 (PDB ID: 5198) were obtained from Protein Data Bank (https://www.rcsb.org/). To prepare the structure for docking, the structure of ligand and proteins were changed into pdbqt format. Autodock vina software was utilized for docking in this study. Optimize the interaction between ligands and receptors via semiflexible docking. In general, the docking parameters remain Vina default but the number of Autodock vina runs was 100 times.

\section{Western blot analysis}

Cell were cultured with the medium containing $0.8 \mathrm{mM}$ TPL or not for $24 \mathrm{~h}$ and rinsed quickly with PBS. Then the cells were lysed with RIPA lysis buffer with $1 \%$ protein phosphatase inhibitors (Fdbio science, Hangzhou, China) and 1\% PMSF (Fdbio science, Hangzhou, China). Protein content was measured using BCA protein kit (Beyotime, Shanghai, China). For measuring the expression level and phosphorylation level of IDH1/IDH2, $10 \mu \mathrm{g}$ of cell lysates were separated by $10 \%$ SDS-PAGE and $10 \%$ phosbind SDS-PAGE (Apexbio, Houston, TX, USA) respectively. The phosbind SDS-PAGE was washed gently in protein transfer buffer with $5 \mathrm{mmol} / \mathrm{l}$ EDTA for three times and general protein transfer buffer once. Then the samples were transferred to PVDF membrane, and blocked in 5\% BSA. PVDF membranes were incubated in appropriate primary antibodies overnight at $4{ }^{\circ} \mathrm{C}$. These antibodies were anti-IDH1 antibody (Abcam, Cambridge, UK, 1:5000 dilution), anti-IDH2 antibody (Abcam, Cambridge, UK, 1:5000 dilution), and anti-GAPDH antibody (Proteintech, Chicago, IL, USA, 1:10,000 dilution). The membranes were washed with TBST and then incubated with HRPconjugated secondary antibodies (Bioworld, Minneapolis, MN, USA, 1:10000 dilution) for $1 \mathrm{~h}$. The bands were scanned using Bio-Rad molecular imager.

\section{Enzyme assays}

The activities of IDH1 and IDH2 were detected using Isocitrate Dehydrogenase Activity Colorimetric Assay Kit according to the manufacturer's instructions (BioVision, SanFrancisco, CA, USA). Briefly, recombinant human IDH1 (Abcam, Cambridge, UK) and IDH2 (Abcam, Cambridge, UK) were diluted with IDH assay buffer, and added into 96-well plate. And indicated concentration of TPL were added to these wells. Adjust the final volume to $50 \mu \mathrm{l}$ with assay buffer. A total of $50 \mu \mathrm{l}$ of the reaction mix, containing $\mathrm{NADP}^{+}$, IDH substrate, and developer, was added to each well. The mix was incubated for $3 \mathrm{~min}$ at $37^{\circ} \mathrm{C}$. The OD $450 \mathrm{~nm}$ was measured in a Bio-Tek multi-mode reader, and then the absorbance was read at $450 \mathrm{~nm}$ every $10 \mathrm{~min}$, for 90 mins. The mean values of background wells without enzyme were subtracted from all readings.

\section{Animals}

Female BALB/c nude (nu/nu) mice (5-8-week old) were obtained from the Animal Experimental Center of Guangdong (China). The mice were house in a climate controlled and control 12-h circadian rhythm-adjusted room, and were allowed access to food and water ad libitum. All animal experiments in this study were approved by the Medical Ethics Committee of Southern Medical University.

\section{Tumor and mice models}

SKOV3 cells were implanted subcutaneously (s.c.) in $\mathrm{BALB} / \mathrm{c}$ nude $(\mathrm{nu} / \mathrm{nu})$ mice at a concentration of $2 \times 10^{6}$ in both flanks. Tumor size was assessed every 2 days by caliper measurements, using the formula width ${ }^{2} x$ length $\times 0.5$. Mice received intraperitoneal (i.p.) drug treatment when tumors reached $50-100 \mathrm{~mm}^{3}$. The mice were randomly divided into two groups: $\mathrm{CON}$ (saline, $n=$ 9) and TPL ( $1.6 \mathrm{mmol} / \mathrm{kg}$, daily, $n=9)$. All animals were weighed every day.

\section{Hematoxylin and eosin staining}

Mouse kidneys and livers were harvested 14 days after start of therapy, and fixed in $4 \%$ paraformaldehyde for $24 \mathrm{~h}$, then gradually dehydrated and embedded in paraffin. The samples were cut into $3-\mu \mathrm{m}$ sections and stained with hematoxylin and eosin (H\&E) for general histological examination.

\section{Immunohistochemistry}

Tumors were harvested 21 days after start of therapy. Tumors were fixed in $4 \%$ paraformaldehyde and embedded in paraffin. A total of 3- $\mu$ m thick sections were stained for Ki-67 (Abcam, Cambridge, UK, 1:300 dilution) followed by HRP-conjugated secondary antibody using diaminobenzidine reagents and then counterstained with hematoxylin. Negative control was performed by omitting primary antibody. Five fields per tumor were randomly selected at a magnification of $\times 200$ and $\times 400$. The percentage positive cells were determined for each field.

\section{Tunel assay}

The apoptosis detection in tumor tissue were performed using TUNEL apoptosis detection kit according to the manufacturer's instructions (YEASEN, Shanghai, China). A total of 3- $\mu \mathrm{m}$ thick sections were dewaxed with xylene and gradually dehydrated with concentration gradient alcohol. Tissue sections were incubated with $2 \mathrm{mg} / \mathrm{ml}$ proteinase $\mathrm{K}$ for $20 \mathrm{~min}$ at $\mathrm{RT}$. Then each sample was kept in $100 \mu \mathrm{L} 1 \times$ equilibration buffer for $30 \mathrm{~min}$. A total of 
$2 \mu \mathrm{g} / \mathrm{ml}$ DAPI was used to stain samples for $5 \mathrm{~min}$ after incubating with $50 \mu \mathrm{TdT}$ incubation buffer for $60 \mathrm{~min}$ at $37^{\circ} \mathrm{C}$ away from light, and examined using a fluorescent microscope.

\section{Statistical analysis}

Results are presented as mean \pm standard deviation. For pairwise comparisons, statistical analysis was performed using the Student's $t$-test, and the statistical analyses were performed using GraphPad Prism statistical software (GraphPad Software, Inc., San Diego, CA, USA). Differences in tumor growth at specific time points were analyzed by one-way ANOVA, and these analyses were performed with the SPSS statistical software (SPSS, Inc. Chicago, IL, USA). $P$ value $<0.05$ was considered statistically significant. Statistical significance was defined as ${ }^{*} P<0.05 ;{ }^{* *} P<0.01 ;{ }^{* * *} P<0.001$; no sign, no significant difference.

\section{Results \\ TPL treatment reduces ROS level and inhibits mitochondrial OXPHOS}

We detected the cell proliferation after the treatment of TPL and we observed that $1 \mathrm{mM}$ TPL treatment for $48 \mathrm{~h}$ significantly reduced cell proliferation (Fig. 1a), while cellular morphology hasn't observable change (data not show). ROS is critical for the effectiveness of chemotherapeutic drugs. We analyzed cellular ROS by using emission fluorescence intensity in cells incubated in the presence of DCFH-DA and found that cellular ROS level was increased in a concentration-dependent matter when the concentration of TPL was $>2 \mathrm{mM}$ (Fig. 1b), while the ROS level was not increased by lower concentration TPL treatment $(1 \mathrm{mM})$, suggesting that the effect of TPL on ROS production in cancer cells depended on the concentration of TPL (Table 1).

Mitochondrial is a major source of ROS, and TPL was reported to reduce the cellular OXPHOS in zebrafish ${ }^{29}$. To determine the effect of TPL on OXPHOS in cancer cells, we used extracellular flux analyzer Seahorse XF96e to measure the OCR. After the 24-h treatment of TPL, basal mitochondrial OCR was significantly reduced in SKOV3 (Fig. 1c, d). After treating with oligomycin and protonophoric uncoupler FCCP, both maximal and reserve mitochondrial capacities were significantly reduced in the presence of TPL. Besides, the proton leak and the non-mito oxygen consumption (using the electron transport inhibitor rotenone) were reduced by TPL treatment. Next, we evaluated the baseline of OCR in cells treated with indicated concentration of TPL and showed that the baseline of OCR was significantly reduced (Fig. 1e), in line with the above result. These results indicated that TPL treatment inhibited OXPHOS of SKOV3 cells.
We then detected the content of NADH that is generated mainly from the TCA cycle and donates electrons for complex I as part of OXPHOS in cells. The data showed that TPL treatment significantly increased the content of $\mathrm{NAD}^{+}$and elevated the ratio of $\mathrm{NAD}^{+} / \mathrm{NADH}$ (Fig. 2a, b), suggesting that TPL treatment inhibited the production of NADH from the TCA cycle.

\section{The effect of TPL on glycolysis in cancer cells}

The disturbance of the TCA cycle is an important cause of mitochondrial respiration inhibition. Glycolysis is critical for generating pyruvate to fuel TCA. To determine whether TPL influences glycolysis of cancer cells, we did GC-MS-based ${ }^{13} \mathrm{C}$ metabolic flux analysis to analyze metabolic fluxes. Mass isotopomer distributions represent the relative abundance of ion fragments with a different number of ${ }^{13} \mathrm{C}$ (ref. ${ }^{30}$ ). Mass isotopomers M0, M1, M2, etc. refers to the ion fragments with zero, one, or two ${ }^{13} \mathrm{C}$, respectively. The steady levels of metabolite and the metabolic pathways by testing the ${ }^{13} \mathrm{C}$-labeled metabolites enable us to analyze metabolic flux distribution (Fig. 3a). SKOV3 cells maintained in media with $\left[\mathrm{U}_{-}{ }^{13} \mathrm{C}_{6}\right]$ glucose was treated or not with $0.8 \mathrm{mM}$ TPL for $24 \mathrm{~h}$ and then were analyzed with GC-MS. The TPL-treated cells exhibited a threefold decrease of total pyruvate production, but no change of total lactate and three amino acids (alanine, glycine, and serine) amount (Fig. 3b). We found that glucose-derived M3 pyruvate, M3 alanine, M3 serine, and M2 glycine but not M3 lactate were significantly reduced in TPL-treated cells when we measured the metabolites labeled with ${ }^{13} \mathrm{C}$ (Fig. 3c). The result indicated that TPL inhibited pyruvate production and serine pathway.

We further evaluated the ECAR and showed that TPL treatment had no effect on extracellular lactate derived from glycolysis and from non-glycolysis (Fig. 3e, f). Our data shows the conversion from pyruvate to lactate increased in TPL-treated cells (Fig. 3d). We also measured glucose consumption of SKOV3 cells treated with TPL by using Glucose Colorimetric Assay. The result showed that TPL treatment didn't change glucose consumption (Fig. 3g). Taken all together, the data suggested that TPL treatment had less effect on glycolysis but reduced the production of pyruvate and inhibited the serine pathway.

\section{TPL interferes with glutamine metabolism by inhibiting conversion between isocitrate and a-ketoglutarate}

We further explored the effect of TPL on cellular metabolism, we analyzed the relative abundance of TCA cycle metabolites derived from glucose. Some TCA cycle intermediates in TPL-treated cells increased significantly and three TCA-related amino acids (aspartate, glutamine, and glutamate) reduced (Fig. 4a). TCA cycle intermediates labeled with M2 isotopomer metabolites $\left({ }^{13} \mathrm{C}_{2}\right.$ citrate, 


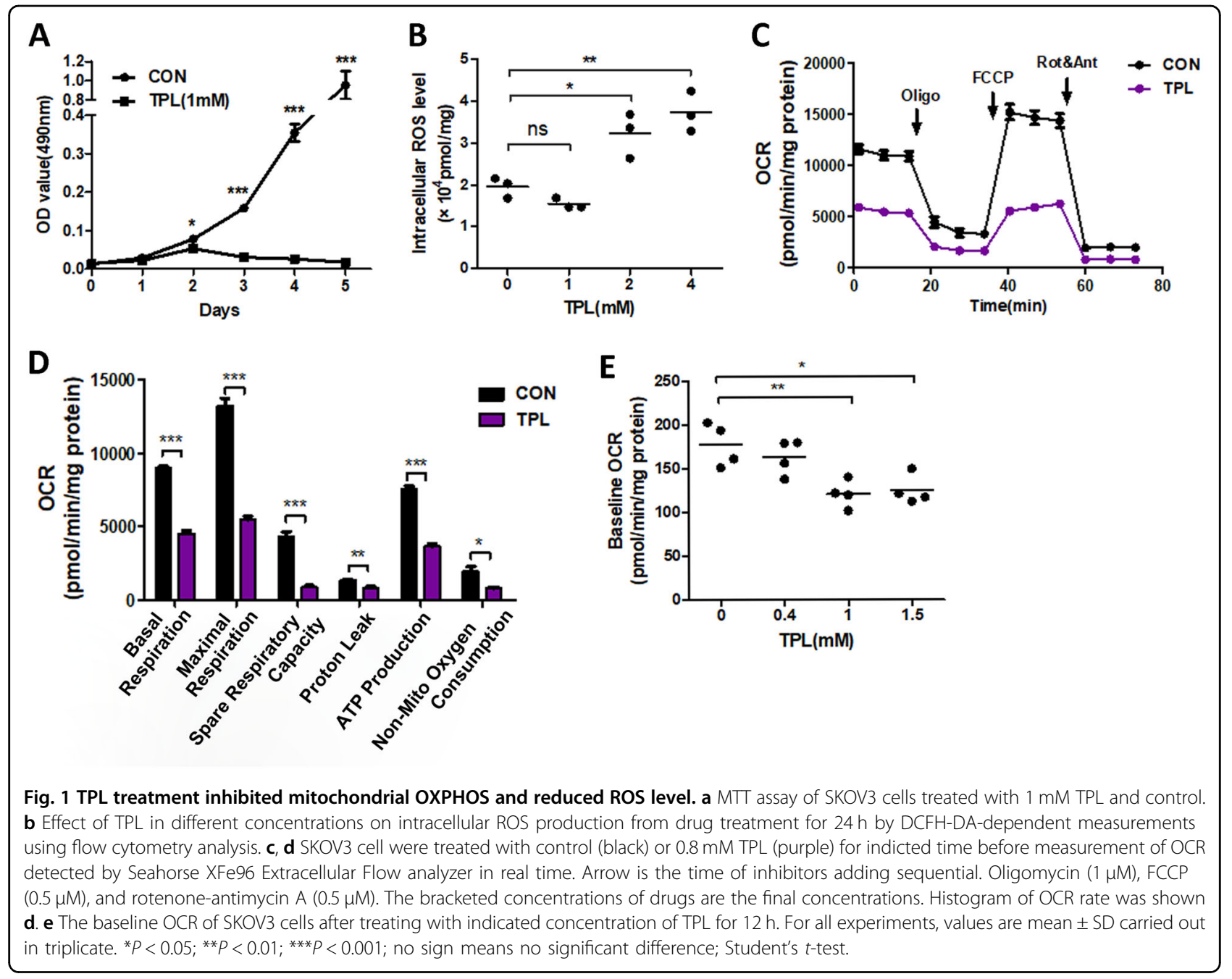

Table 1 Docking results of TPL on both IDH1 and IDH2.

\begin{tabular}{lll}
\hline Protein & PDB ID & $\begin{array}{l}\text { Tempol banding } \\
\text { energy(kcal/mol) }\end{array}$ \\
\hline $\mathrm{IDH} 1$ & $6 \mathrm{ADG}$ & -8.9 \\
$\mathrm{IDH} 2$ & 5198 & -12.7 \\
\hline
\end{tabular}

${ }^{13} \mathrm{C}_{2}$ a-ketoglutarate, ${ }^{13} \mathrm{C}_{2}$ malate, and ${ }^{13} \mathrm{C}_{2}$ glutamate) were derived from $\mathrm{U}_{-}{ }^{13} \mathrm{C}_{6}$ glucose. The percentage of $\mathrm{M} 2$ citrate was significantly increased, but the M2 glutamate (derived from M2 $\alpha$-ketoglutarate), M2 malate, and M2 aspartate (derived from M2 oxaloacetate) were all significantly reduced in TPL-treated SKOV3 cells (Fig. 4b). The ratio of M2 citrate to M3 pyruvate increased significantly, but M2 succinate/M2 citate decreased and not in the M2 malate/M2 succinate ratio after TPL treatment (Fig. 4c), suggesting reducing the effect of TPL on the
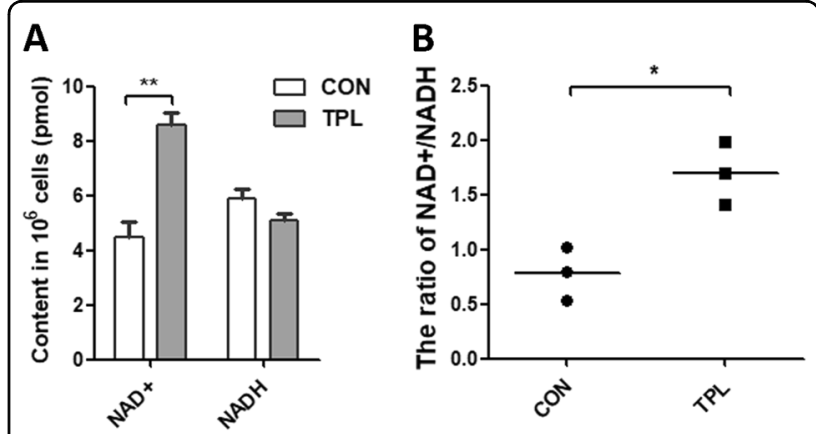

Fig. 2 TPL treatment increased the ratio of $\mathrm{NAD}^{+} / \mathrm{NADH}$ in cells. a The content of $\mathrm{NAD}^{+}$and $\mathrm{NADH}$ in cells after treating with $0.8 \mathrm{mM}$ TPL or not. $\mathbf{b}$ The ratio of $\mathrm{NAD}^{+} / \mathrm{NADH}$ in cells after treating with $0.8 \mathrm{mM}$ TPL or not $(n=3)$. For all experiments, values are mean \pm SD carried out in triplicate. ${ }^{*} P<0.05 ;{ }^{* *} P<0.01 ;{ }^{* *} P<0.001$; no sign means no significant difference; Student's t-test.

oxidation procession from citrate to succinate. The above results indicated that TPL inhibited TCA in the glucose metabolism of cancer cells. 

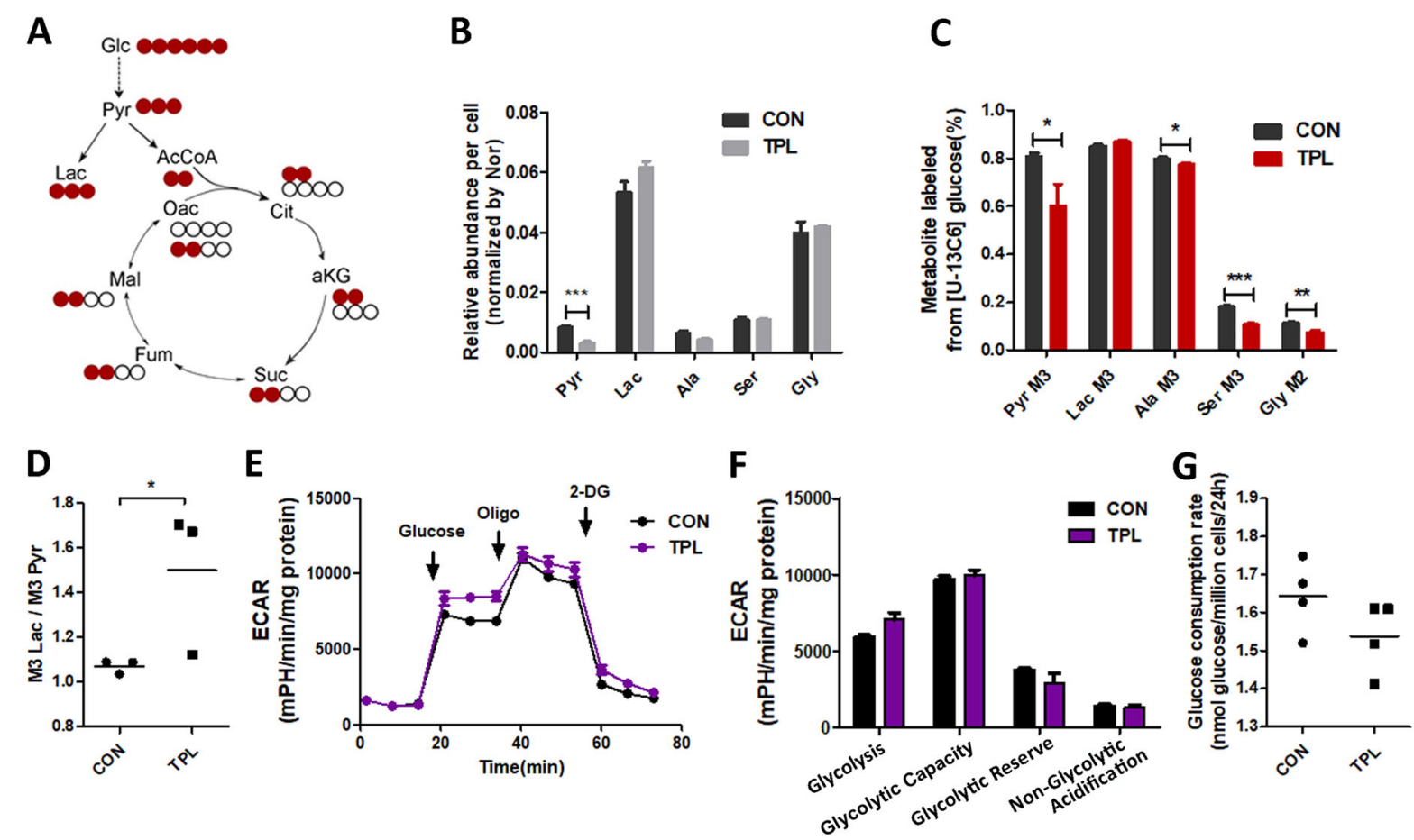

Fig. 3 TPL reduced the production of pyruvate and inhibited the serine pathway. a Schematic map of carbon atom (represented by circles) transitions and tracer used to detect glucose metabolism, $\left[\mathrm{U}_{-}{ }^{13} \mathrm{C}_{6}\right]$ glucose $(\mathrm{red})$. $\mathbf{b}$ Relative abundance of pyruvate, lactate, and three amino acids (alanine, glycine, and serine) per cell after maintaining in $\left[\mathrm{U}^{13} \mathrm{C}_{6}\right]$ glucose medium that contained $0.8 \mathrm{mM}$ TPL (gray) or not (black) for $24 \mathrm{~h}(n=3)$. c Relative level of glycolysis and serine pathway, determined by M3 or M2 labeling of metabolites from $\left[\mathrm{U}_{-}{ }^{13} \mathrm{C}_{6}\right]$ glucose in control (black) and TPL group (red; $n=3$ ). $\mathbf{d}$ The ratios of M3 lactate/M3 pyruvate. e, $\mathbf{f}$ SKOV3 cell were treated with control (black) or $0.8 \mathrm{mM} \mathrm{TPL} \mathrm{(purple)} \mathrm{for} \mathrm{indicted} \mathrm{time}$ before measurement of ECAR were detected by Seahorse XFe Extracellular Flow analyzer in real time $(n=3)$. Arrow indicate the time of inhibitors adding sequential. Glucose $(10 \mathrm{mM})$, oligomycin $(1 \mu \mathrm{M})$, and 2-DG $(50 \mathrm{mM})$; the bracketed concentrations of drugs are the final concentrations. Histogram of ECAR rate was shown $\mathbf{f}$. $\mathbf{g}$ Glucose consumption rate in SKOV3 cells after treated with control (white) or TPL (black) for $24 \mathrm{~h}$ ( $n=4$ ). For all experiments, values are mean $\pm \mathrm{SD}$ carried out in triplicate. ${ }^{*} P<0.05$; ${ }^{* *} P<0.01$; ${ }^{* * *} P<0.001$; no sign means no significant difference; Student's t-test.

We further investigated the effect of TPL on the TCA cycle by GC-MS metabolic flux analysis with $\left[\mathrm{U}_{-}{ }^{13} \mathrm{C}_{5}\right]$ glutamine (Fig. $4 \mathrm{~d}$ ). In glutaminolysis, glutamine is converted into glutamate and then oxidized into $\alpha-\mathrm{KG}$ for TCA metabolism. The abundance of M5 glutamine didn't change in TPL-treated cells (Fig. 4e), indicating TPL didn't increase the uptake of glutamine. The M5 glutamate abundance increased while the M5 $\alpha$-ketoglutarate reduced significantly, leading to a significant reduction for the ratio of M5 glutamate/M5 $\alpha$-ketoglutarate (Fig. 4f). Furthermore, we examined the effect of TPL on cell viability when SKOV3 cells were cultured with glutaminefree medium supplementation with $\alpha$-ketoglutarate. The rate of Gln-free + Akg + TPL/Gln-free + Akg had an increase compared with the rate in the medium without $\alpha$-ketoglutarate $(P<0.05$, Fig. $4 \mathrm{~g})$, indicating that the inhibitory effect of TPL on cell viability was partially rescued after $\alpha$-ketoglutarate supplementation. This result suggests that inhibition of either transaminases or GDH and conversion of glutamate to $\alpha$-ketoglutarate was impeded after TPL treatment.
After entering to TCA cycle, M5 $\alpha$-ketoglutarate converted into succinate, fumarate, malate, and citrate labeled with M4 isotopomer metabolites $\left({ }^{13} \mathrm{C}_{4}\right.$ succinate, ${ }^{13} \mathrm{C}_{4}$ fumarate, ${ }^{13} \mathrm{C}_{4}$ malate, and ${ }^{13} \mathrm{C}_{4}$ citrate). As showed in Fig. 4h, TPL induced an increase of M5 glutamine-derived oxidative intermediates M4 malate, and M4 citrate, but the reduction of M2 succinate from M4 citrate (Fig. 4h). The ratio of M3 $\alpha$-ketoglutarate/M4 citrate was reduced but the ratio of M4 fumarate/M4 succinate, M4 malate/ M4 fumarate, M4 citrate/M4 malate, and M2 succinate/ M3 $\alpha$-ketoglutarate didn't change or even slightly increased (Fig. 4i, j). Accumulation of M4 intermediates may explain the impeding procession of citrate to $\alpha$ ketoglutarate.

Glutamine can also generate acetyl-CoA via reductive carboxylation, wherein glutamine-derived $\alpha$-ketoglutarate is carboxylated to produce isocitrate/citrate, which is then cleaved to produce oxaloacetate and acetyl-CoA. In the GC-MS FMA assay, $\left[\mathrm{U}-_{-}^{13} \mathrm{C}_{5}\right]$ glutamine-derived M5 $\alpha$-ketoglutarate is carboxylated to produce to M5 citrate and further decarboxylated to other 4-carbon 


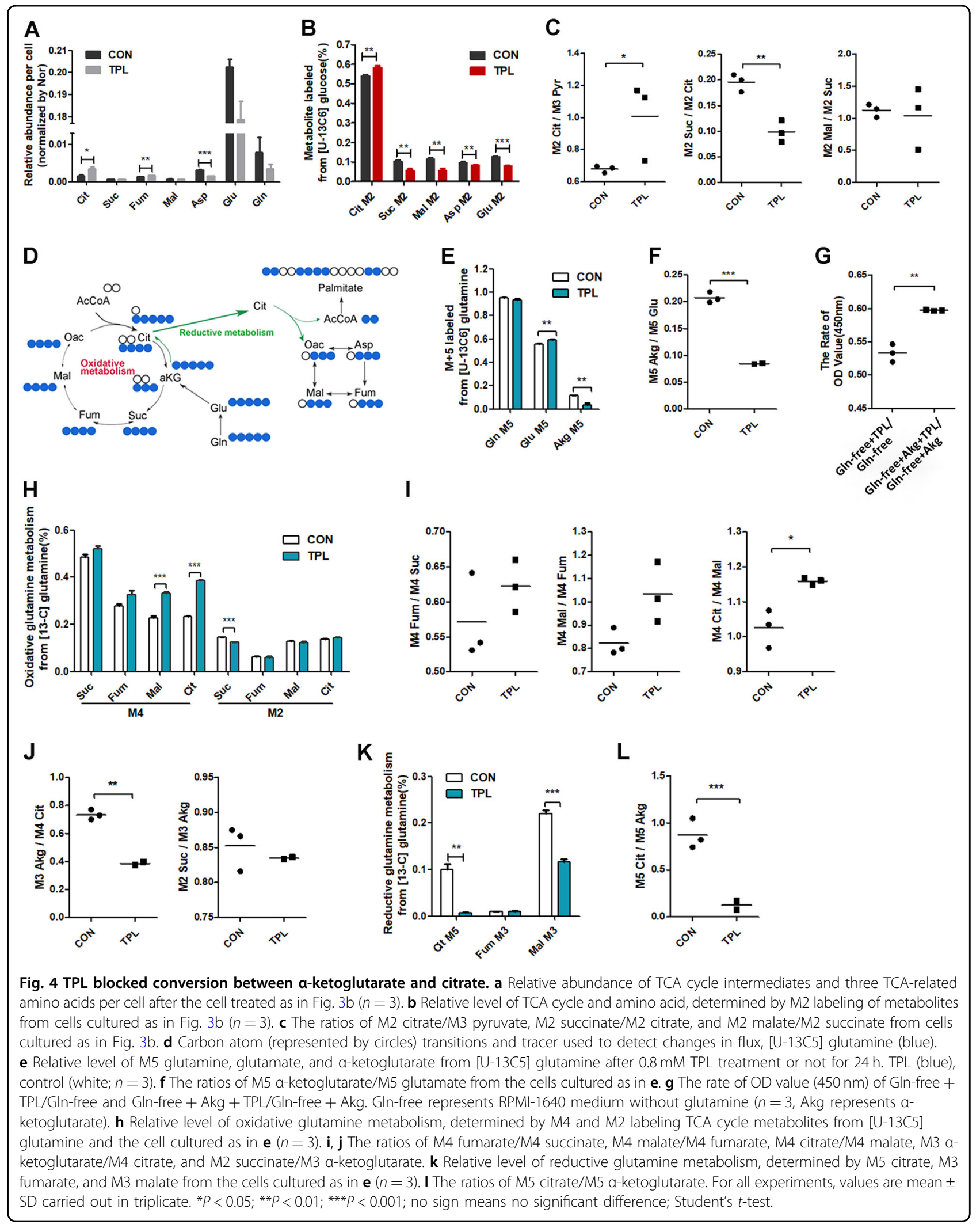



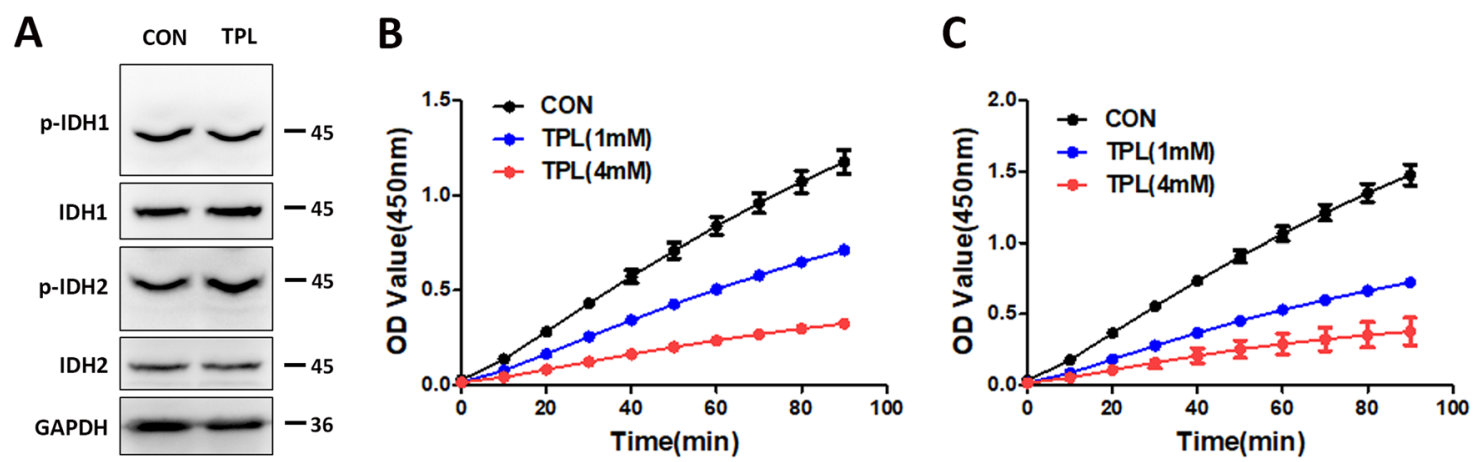

Fig. 5 TPL inhibited the IDH1 and IDH2 enzyme activity. a The effects of TPL on the phosphorylation of IDH1 and IDH2 in SKOV3 cells were analyzed by a Phos-tag assay. Immunoblots for total IDH1 and IDH2 were also performed on normal SDS-PAGE gels. $\mathbf{b}, \mathbf{c}$ Enzymatic activity of both IHD1 and IDH2 treated with control and TPL $(n=3)$. For all experiments, values are mean \pm SD carried out in triplicate. ${ }^{*} P<0.05 ;{ }^{* *} P<0.01 ;{ }^{* * *} P<$ 0.001 ; no sign means no significant difference; Student's t-test.

intermediates (M3 malate, M3 fumarate, M3 oxaloacetate, and M3 aspartate). The abundance of the M5 citrate was dramatically reduced in TPL-treated SKOV3 cells and M3 malate also reduced onefold (Fig. 4k). To eliminate the effect of the decrease abundance of $\alpha$-ketoglutarate, we evaluated the amount of M5 citrate by normalizing the amount of M5 $\alpha$-ketoglutarate in both groups. The ratio of M5 citrate/M5 $\alpha$-ketoglutarate was significantly decreased after TPL treatment (Fig. 4l), suggesting that TPL blocks the conversion of $\alpha$-ketoglutarate to isocitrate. Taken it together, the results indicated that TPL interferes with glutamine metabolism by blocking conversion between isocitrate and $\alpha$-ketoglutarate.

\section{TPL inhibited the activity of IDH1 and IDH2 directly in SKOV3 cells}

Conversion between citrate and $\alpha$-ketoglutarate is a two-step reaction, including the conversion of citrate-toisocitrate and isocitrate-to- $\alpha$-ketoglutarate. The conversion of isocitrate-to- $\alpha$-ketoglutarate is a key step that is catalyzed by IDHs, most frequently mutated proteins in cancer $^{31,32}$. There are three IDH isoforms, of which IDH1 and IDH2 catalyze either oxidative decarboxylation or reduction carboxylation ${ }^{33,34}$. To investigate whether TPL inhibited conversion between $\alpha$-ketoglutarate and citrate by inhibiting IDH1/2 expression and phosphorylation, we performed western blotting. The result showed that the TPL treatment didn't change IDH1/2 expression and phosphorylation (Fig. 5a).

To clarify the ability of TPL binding to the target protein and identify the binding mode, we conducted docking simulations for TPL to IDH1/2 by applied Autodock vina. The result shows the binding energies of TPL to IDH1 and IDH2 are -8.9 and $-12.7 \mathrm{kcal} / \mathrm{mol}$, respectively (Table 1). We then determined the activities of recombinant IDH1/2 by detection of the increase in NADPH concentration. The results showed that TPL treatment directly inhibit the activity of purified recombinant IDH1/ 2 protein. These results indicated that TPL inhibited IDH1/2 activity directly but not inhibiting IDH1/2 expression and phosphorylation (Fig. 5b, c).

\section{TPL inhibited the growth of SKOV3 cells in xenograft animal model}

To examine whether TPL has antitumor activity in a mouse model, we established a xenograft mouse model by s.c. injection of SKOV3 cells to BALB/c nude mice. We applied a dose of TPL to $1.6 \mathrm{mmol} / \mathrm{kg}$, a significant inhibition of tumor growth was observed and the lifespan was prolonged although it's not significant in statistics (Fig. 6a, b), indicating that TPL treatment inhibiting tumor growth in xenografted mice. The TPL-treated group also displayed no effect on body weight and histo-morphology of kidney and liver analyzed by HE staining (Fig. 6c). The cell proliferation was detected by the expression of Ki-67 in tumor tissues. The percentages of the Ki-67-positive cell in tumor tissues decreased in TPL-treated mice (Fig. 6d).

The elevated oxidative stress is an important inducer of apoptosis. We then detected whether TPL had an inhibitory effect on tumor cell apoptosis in the mouse model. TUNEL assay showed that TPL markedly reduced the number of apoptotic cells compared with control (Fig. 6e). These results demonstrate that the inhibitory effect of TPL on tumor growth was not by inducing cell apoptosis, but by inhibiting cell proliferation in vivo in the xenograft tumor model.

\section{Discussion}

TPL has been reported to induce cellular apoptosis of many types of cancer cells and inhibit tumor growth in immune-defective mice ${ }^{35,36}$. In this study, we confirmed that the cytotoxicity of TPL was dependent on high concentration and induced cellular apoptosis that associated to increase in cellular ROS. However, the dosage of 


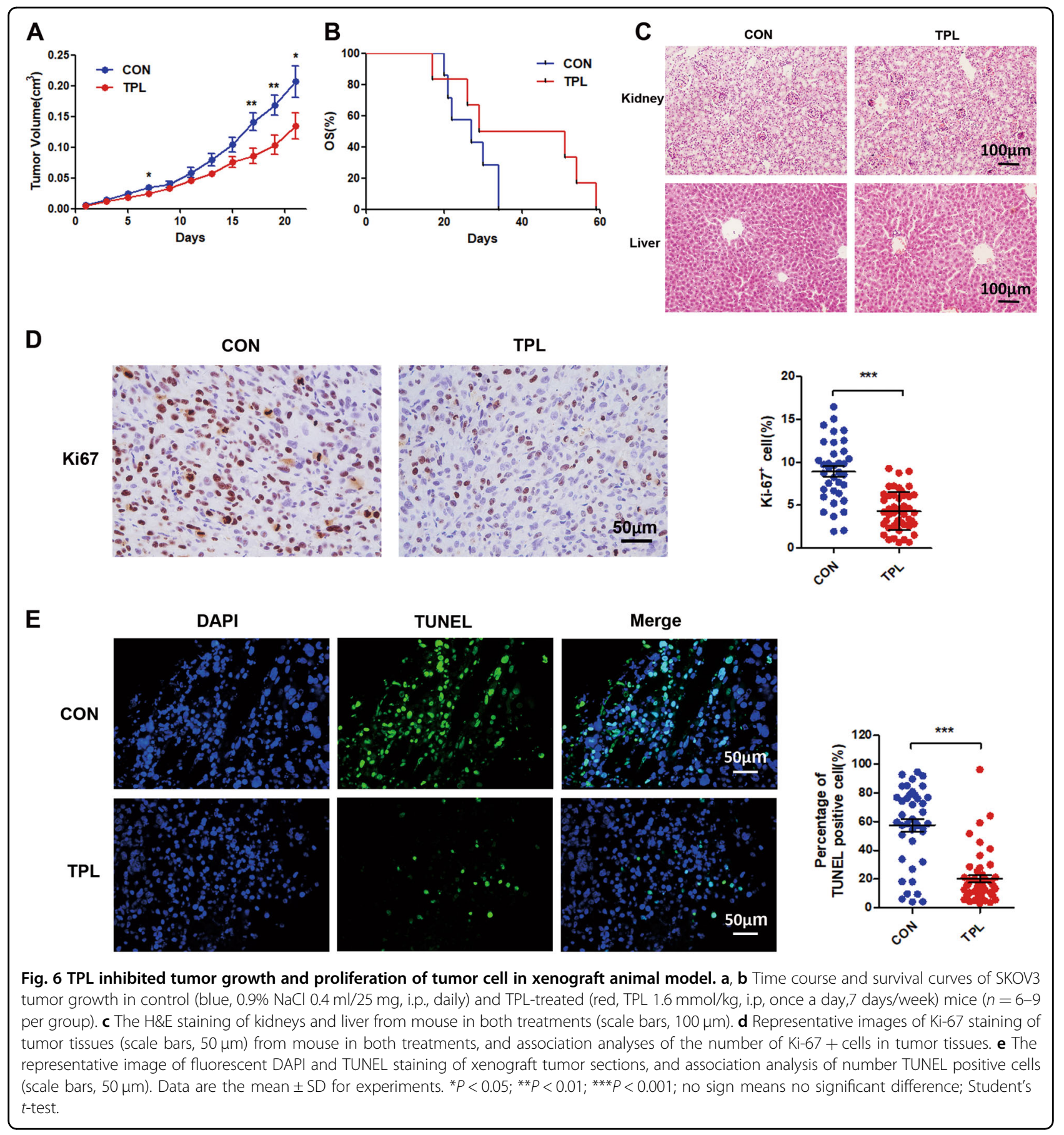

TPL used in vivo inhibited cellular proliferation but not increase the events of cellular apoptosis. The concentration of TPL in the tumors of xenograft mice cannot reach the concentration of inducing ROS, so it may work as an antioxidant in vivo. Here, we proposed a new mechanism of TPL inhibiting cancer cell proliferation by interfering with glutamine metabolism.

Cellular proliferation is closely associated with metabolism. The rapid proliferation of cancer cells requires a large number of macromolecular precursors and NADPH produced from glycolysis and TCA cycle ${ }^{33,37,38}$. It is well known that, in addition to glucose, glutamine is also an important nutrient for tumor cell growth and viability ${ }^{23}$. Glutamine is a conditionally essential amino acid, and can provide nitrogen and carbon for the TCA cycle, fatty acid synthesis and nucleotide synthesis ${ }^{34,39,40}$. Glutamine metabolism includes the catabolic process (glutaminolysis) and anabolic process (reductive carboxylation), 
which are vital for many cancer cells to maintain survival. Glutaminolysis can fuel the oxidative TCA cycle flux, which is important for sustaining ATP production and redox homeostasis in cells, especially when glucose preferentially enters the process of aerobic glycolysis in cancer cells ${ }^{41-43}$. Glutamine also provides the major source of acetyl-CoA for lipid synthesis via reductive carboxylation, which significantly reduces the need for acetyl-CoA derived from glucose. Furthermore, the citrate produced by the reductive carboxylation is cleaved to oxaloacetate, which can be converted to other 4-carbon intermediates to complement the TCA cycle ${ }^{22,44}$. Besides, it is reported that blocking glutamine can relieve the immunosuppressive effect of the tumor microenvironment, and at the same time can enhance the activity of $\mathrm{T}$ cells ${ }^{45}$. Hence, disturbing glutamine metabolism can be a strategy for cancer treatment.

Here, we found that TPL had no significant effect on glycolysis although it lowered the production of pyruvate and intermediates of the serine pathway. However, TPL not only blocked the generation of $\alpha$-ketoglutarate from glutamate but also significantly interfered with both glutaminolysis and reductive carboxylation processes via the inhibition of conversion between citrate/isocitrate and $\alpha$ ketoglutarate, which are catalyzed by IDHs.

There are three isoforms of IDH in a human cell: IDH1, IDH2, and IDH3. IDH3 uses $\mathrm{NAD}^{+}$as cofactor and functions exclusively as oxidative decarboxylase. IDH1 and IDH2 catalyze either oxidative decarboxylation or reduction carboxylation using $\mathrm{NADP}^{+} / \mathrm{NADPH}$ as cofactors $^{34,43}$. Recent studies suggest that IDH1 and IDH2 mutations exist in a variety of tumor types, such as glioma, chondrosarcoma, and acute myeloid leukemia ${ }^{46}$. Besides, the aberrant expression of wild-type IDHs is also found in some tumors. Overexpression of IDHs is associated with the poor overall survival of cancer patients ${ }^{47-49}$. Our results suggested that the inhibition of TPL on activities of IDH1/IDH2 to interfere with the interconversion between citrate and $\alpha$-ketoglutarate in TCA cycle is one important mechanism of TPL disturbing cellular metabolism. Blocking citrate metabolism by TPL led to the citrate accumulation and downstream metabolites reduction. First, citrate is an allosteric inhibitor of phosphofructokinase (PFK), a key enzyme regulating glycolysis flow in tumor cells ${ }^{50}$. Accumulation of citrate inhibits the activity of PFK, which may explain reduced glucose-derived pyruvate production and inhibited serine pathway in TPL-treated cells. Second, the TCA cycle generates a large number of $\mathrm{NADH} / \mathrm{FADH}_{2}$, which provides electrons for OXPHOS (ref. ${ }^{51}$ ). TPL inhibited the conversion of citrate to $\alpha$-ketoglutarate and disturbed the OXPHOS at complex I by inhibiting NADH production in cells. These results were consistent with the previous reports, in which TPL impaired complex I in oxidation respiratory chain. Third, TPL perturbing glutamine metabolism could further inhibit the TCA cycle and lipid synthesis, which may explain the reduced cellular proliferation and slower growth of tumors after TPL treatment.

Collectively, TPL exerts multiple mechanisms to influence cell viability. Here, we provided a mechanism of TPL inhibiting cancer cell proliferation by interfering with glutamine utilization, which is important for survival and proliferation of cancer cells. The study may help the development of a therapeutic strategy of TPL combined with other anticancer medicines.

\section{Acknowledgements}

This work was supported by the National Natural Science Foudation of China (81472834 to Q. L., 31670892 to B.H., and 61427807 to W.H.).

\section{Author details}

${ }^{1}$ Cancer Research Institute, Guangdong Provincial Key Laboratory of Cancer Immunotherapy, Guangzhou Key Laboratory of Tumor Immunology Research, School of Basic Medical Sciences, Southern Medical University, Guangzhou, China. ${ }^{2}$ School of Biomedical Engineering, Southern Medical University, Guangzhou, China. ${ }^{3}$ The First Affiliated Hospital of Jinan University, Guangzhou, China. ${ }^{4}$ Zhongshan School of Medicine, Sun Yat-Sen University, Guangzhou, China. ${ }^{5}$ Guangzhou Hospital of integrated Traditional and West Medicine, Guangzhou, China. ${ }^{6}$ Center for medical transformation, Shunde Hospital, Southern Medical University, Foshan, China. ${ }^{7}$ National Key Discipline of Human Anatomy, School of Basic Medical Sciences, Southern Medical University, Guangzhou, China. ${ }^{8}$ Department of Human Anatomy, School of Basic Medical Sciences, Guangdong Medical University, Guangzhou, China

Conflict of interest

The authors declare that they have no conflict of interest.

\section{Publisher's note}

Springer Nature remains neutral with regard to jurisdictional claims in published maps and institutional affiliations.

Received: 22 October 2019 Revised: 5 April 2020 Accepted: 6 April 2020 Published online: 04 May 2020

\section{References}

1. Rossi, S., Giuntini, A., Balzi, M., Becciolini, A. \& Martini, G. Nitroxides and malignant human tissues: electron spin resonance in colorectal neoplastic and healthy tissues. Biochim. Biophys. Acta 1472, 1-12 (1999).

2. Gariboldi, M. B., Rimoldi, V., Supino, R., Favini, E. \& Monti, E. The nitroxide tempol induces oxidative stress, p21(WAF1/CIP1), and cell death in HL60 cells. Free Radic. Biol. Med. 29, 633-641 (2000).

3. Ravizza, R., Gariboldi, M. B., Passarelli, L. \& Monti, E. Role of the p53/p21 system in the response of human colon carcinoma cells to Doxorubicin. BMC Cancer 4, 92 (2004).

4. Lewandowski, M. \& Gwozdzinski, K. Nitroxides as antioxidants and anticancer drugs. Int. J. Mol. Sci. 18, 2490 (2017).

5. Gariboldi, M. B. et al. Antiproliferative effect of the piperidine nitroxide TEMPOL on neoplastic and nonneoplastic mammalian cell lines. Free Radic. Biol. Med. 24, 913-923 (1998).

6. Gariboldi, M. B. et al. The nitroxide Tempol modulates anthracycline resistance in breast cancer cells. Free Radic. Biol. Med. 40, 1409-1418 (2006).

7. Monti, E. et al. Nitroxide TEMPOL impairs mitochondrial function and induces apoptosis in HL60 cells. J. Cell. Biochem. 82, 271-276 (2001).

8. Alpert, E. et al. 4-Hydroxy tempol-induced impairment of mitochondrial function and augmentation of glucose transport in vascular endothelial and smooth muscle cells. Biochem. Pharmacol. 67, 1985-1995 (2004). 
9. D, V., A, A. \& P, N. Platinum Complexes with Bioactive Nitroxyl Radicals: Synthesis and Antitumor Properties. (Intech, Croatia, 2012).

10. Pattison, D. I., Lam, M., Shinde, S. S., Anderson, R. F. \& Davies, M. J. The nitroxide TEMPO is an efficient scavenger of protein radicals: cellular and kinetic studies. Free Radic. Biol. Med. 53, 1664-1674 (2012).

11. De Blasio, M. J. et al. The superoxide dismutase mimetic tempol blunts diabetes-induced upregulation of NADPH oxidase and endoplasmic reticulum stress in a rat model of diabetic nephropathy. Eur. J. Pharmacol. 807, 12-20 (2017).

12. Bernardy, C. C. F. et al. Tempol, a superoxide dismutase mimetic agent, inhibits superoxide anion-induced inflammatory pain in mice. BioMed. Res. Int. 2017, 9584819 (2017).

13. Dornas, W. C. et al. Oxidative stress causes hypertension and activation of nuclear factor-kappaB after high-fructose and salt treatments. Sci. Rep. 7, 46051 (2017).

14. Obrosov, A., Shevalye, H., Coppey, L. J. \& Yorek, M. A. Effect of tempol on peripheral neuropathy in diet-induced obese and high-fat fed/low-dose streptozotocin-treated C57Bl6/J mice. Free Radic. Res. 51, 360-367 (2017).

15. Liu, Y. et al. Renal and cerebral RAS interaction contributes to diabetic kidney disease. Am. J. Transl. Res. 11, 2925-2939 (2019).

16. Alzoubi, K. H., Khabour, O. F., Albawaana, A. S., Alhashimi, F. H. \& Athamneh, R. Y. Tempol prevents chronic sleep-deprivation induced memory impairment. Brain Res. Bull. 120, 144-150 (2016).

17. Sun, L. et al. Inhibition of NOX2-NLRP1 signaling pathway protects against chronic glucocorticoids exposure-induced hippocampal neuronal damage. Int. Immunopharmacol. 74, 105721 (2019).

18. Zhang, G. et al. Protective effect of tempol on acute kidney injury through PI3K/Akt/Nrf2 signaling pathway. Kidney Blood Press. Res. 41, 129-138 (2016).

19. Vander Heiden, M. G. \& DeBerardinis, R. J. Understanding the intersections between metabolism and cancer biology. Cell 168, 657-669 (2017).

20. Owen, O. E., Kalhan, S. C. \& Hanson, R. W. The key role of anaplerosis and cataplerosis for citric acid cycle function. J. Biol. Chem. 277, 30409-30412 (2002).

21. Garcia-Diaz, D. F. et al. Vitamin C inhibits leptin secretion and some glucose/ lipid metabolic pathways in primary rat adipocytes. J. Mol. Endocrinol. 45 33-43 (2010).

22. DeBerardinis, R. et al. Beyond aerobic glycolysis: transformed cells can engage in glutamine metabolism that exceeds the requirement for protein and nucleotide synthesis. Proc. Natl Acad. Sci. USA 104, 19345-19350 (2007).

23. Altman, B. J., Stine, Z. E. \& Dang, C. V. From Krebs to clinic: glutamine metabolism to cancer therapy. Nat. Rev. Cancer 16, 619-634 (2016).

24. Wise, D. R. et al. Myc regulates a transcriptional program that stimulates mitochondrial glutaminolysis and leads to glutamine addiction. Proc. Natl Acad. Sci. USA 105, 18782-18787 (2008)

25. Reitzer, L. J., Wice, B. M. \& Kennell, D. Evidence that glutamine, not sugar, is the major energy source for cultured HeLa cells. J. Biol. Chem. 254, 2669-2676 (1979).

26. Salvi, A., Patki, G., Khan, E., Asghar, M. \& Salim, S. Protective effect of tempol on buthionine sulfoximine-induced mitochondrial impairment in hippocampal derived HT22 cells. Oxid. Med. Cell. Longev. 2016, 5059043 (2016).

27. Ranjbar, A. et al. Antioxidative effects of tempol on mitochondrial dysfunction in diabetic nephropathy. Iran. J. Kidney Dis. 12, 84-90 (2018).

28. Liu, Y. et al. Tempol protects cardiomyocytes from nucleoside reverse transcriptase inhibitor-induced mitochondrial toxicity. Toxicol. Sci. 139, 133-141 (2014).

29. Jia, J. et al. Reactive oxygen species participate in liver function recovery during compensatory growth in zebrafish (Danio rerio). Biochem. Biophys. Res. Commun. 499, 285-290 (2018).
30. Zamboni, N. 13C metabolic flux analysis in complex systems. Curr. Opin. Biotechnol. 22, 103-108 (2011).

31. Arai, M., Nobusawa, S., Ikota, H., Takemura, S. \& Nakazato, Y. Frequent IDH1/2 mutations in intracranial chondrosarcoma: a possible diagnostic clue for its differentiation from chordoma. Brain Tumor Pathol. 29, 201-206 (2012).

32. Wang, H. Y. et al. The comparison of clinical and biological characteristics between IDH1 and IDH2 mutations in gliomas. J. Exp. Clin. Cancer Res. 35, 86 (2016).

33. Hirschey, M. D. et al. Dysregulated metabolism contributes to oncogenesis. Semin. Cancer Biol. 35, S129-s150 (2015).

34. Mullen, A. R. et al. Reductive carboxylation supports growth in tumour cells with defective mitochondria. Nature 481, 385-388 (2011).

35. Suy, S., Mitchell, J. B., Samuni, A., Mueller, S. \& Kasid, U. Nitroxide tempo, a small molecule, induces apoptosis in prostate carcinoma cells and suppresses tumor growth in athymic mice. Cancer 103, 1302-1313 (2005).

36. Gariboldi, M. B. et al. Study of in vitro and in vivo effects of the piperidine nitroxide Tempol - a potential new therapeutic agent for gliomas. Eur. J. Cancer 39, 829-837 (2003).

37. Schulz, T. J. et al. Glucose restriction extends Caenorhabditis elegans life span by inducing mitochondrial respiration and increasing oxidative stress. Cell Metab. 6, 280-293 (2007).

38. Zhang, J., Pavlova, N. N. \& Thompson, C. B. Cancer cell metabolism: the essential role of the nonessential amino acid, glutamine. EMBO J. 36 1302-1315 (2017)

39. Fan, T. W., Tan, J., McKinney, M. M. \& Lane, A. N. Stable isotope resolved metabolomics analysis of ribonucleotide and RNA metabolism in human lung cancer cells. Metabolomics 8, 517-527 (2012).

40. Yang, L., Venneti, S. \& Nagrath, D. Glutaminolysis: a hallmark of cancer metabolism. Annu. Rev. Biomed. Eng. 19, 163-194 (2017).

41. Le, A. et al. Glucose-independent glutamine metabolism via TCA cycling for proliferation and survival in B cells. Cell Metab. 15, 110-121 (2012).

42. Jiang, L. et al. Reductive carboxylation supports redox homeostasis during anchorage-independent growth. Nature 532, 255-258 (2016).

43. Metallo, C. M. et al. Reductive glutamine metabolism by IDH1 mediates lipogenesis under hypoxia. Nature 481, 380-384 (2011).

44. Yoo, H., Antoniewicz, M. R., Stephanopoulos, G. \& Kelleher, J. K. Quantifying reductive carboxylation flux of glutamine to lipid in a brown adipocyte cell line. J. Biol. Chem. 283, 20621-20627 (2008).

45. Leone, R. D. \& Zhao, L. Glutamine blockade induces divergent metabolic programs to overcome tumor immune evasion. Science 366, 1013-1021 (2019).

46. Bergaggio, E. \& Piva, R. Wild-Type IDH enzymes as actionable targets for cancer therapy. Cancers 11, 563 (2019).

47. Li, J. et al. Wild-type IDH2 promotes the Warburg effect and tumor growth through HIF1alpha in lung cancer. Theranostics 8, 4050-4061 (2018).

48. Al-Amodi, H., Nabih, E. S., Kamel, H. F. M., El Sayed, M. A. \& Dwedar, I. A. M. Wild-type isocitrate dehydrogenase 1 over-expression is related to cancer stem cells survival in lung adenocarcinoma. Cancer Investig. 36, 185-189 (2018).

49. Calvert, A. E. et al. Cancer-associated IDH1 promotes growth and resistance to targeted therapies in the absence of mutation. Cell Rep. 19, 1858-1873 (2017).

50. Kemp, R. G., Fox, R. W. \& Latshaw, S. P. Amino acid sequence at the citrate allosteric site of rabbit muscle phosphofructokinase. Biochemistry $\mathbf{2 6}$ 3443-3446 (1987)

51. Titov, D. V. et al. Complementation of mitochondrial electron transport chain by manipulation of the NAD+/NADH ratio. Science 352, 231-235 (2016). 